\title{
Performance of FlexToT Time Based PET Readout ASIC for Depth of Interaction Measurements
}

\author{
J. Trenado ${ }^{a *}$, J. M. Cela ${ }^{b}$, A. Comerma ${ }^{a}{ }^{\dagger}$ D. Gascon ${ }^{a}$, R. Graciani ${ }^{a}$, L. Freixas ${ }^{b}$, J. \\ Marin $^{b}$, G. Martínez ${ }^{b}$, R. Masachs ${ }^{a}$, J.M. Perez ${ }^{b}$, P. Rato ${ }^{b}$, D. Sanchez ${ }^{a}$, A. Sanuy ${ }^{a}$, I. \\ Sarasola ${ }^{b}$ \\ ${ }^{a}$ University of Barcelona, Spain \\ ${ }^{b}$ CIEMAT, Spain \\ E-mail: jtrenado@ecm.ub.edu, josemanuel.cela@ciemat.es, \\ comerma@physi.uni-heidelberg.de, dgascon@ecm.ub.edu, \\ graciani@ecm.ub.edu, lluis.freixas@ciemat.es, \\ jesus.marin@ciemat.es, gustavo.martinez@ciemat.es, \\ rmasachs@ecm.ub.edu, jm.perez@ciemat.es, pedro.rato@ciemat.es, \\ dsanchez@ecm.ub.edu, asanuy@ecm.ub.edu, iciar.sarasola@ciemat.es
}

\begin{abstract}
The FlexToT ASIC, a read-out electronics for Positron Emission Tomography (PET), has been developed using novel technology, inherited from the field of High Energy Physics (HEP). Using a novel current input stage and a linearized Time over Threshold (ToT) design the FlexToT electronics obtains results within the requirements of the PET technology with simpler and more robust system and using less power consumption.

In this work, the capability of the developed ASIC to resolve depth of interaction (DOI) in PET detectors is tested. Results obtained using an implementation of phoswich detector with two crystals, a GSO and LYSO, show a 99\% of correct identification rate for separated crystals. Measurements with stacked crystals are currently in progress. Preliminary results with this configuration are encouraging.
\end{abstract}

Technology and Instrumentation in Particle Physics 2014,

2-6 June, 2014

Amsterdam, the Netherlands

\footnotetext{
* Speaker.

${ }^{\dagger}$ Current affiliation: Physikalisches Institut - Heidelberg Universität, Germany.
} 


\section{Introduction}

Positron Emission Tomography (PET) [1], depending on the application, can be divided in pre-clinical imaging, organ dedicated imaging and whole-body imaging. Spatial resolution is a key parameter for these applications in the performance of the detectors [6]. Pre-clinical and dedicated organ PET imaging request resolution in the range of $1 \mathrm{~mm}^{3}$ volume resolution. In general, whole body PET imaging can relax this figure

The parallax error is an important degrading factor for the spatial resolution in real PET systems. It becomes relevant when the thickness of the detector is significant and the incident direction of the gamma photon is oblique. Without the estimate of the depth of interaction, uncertainties in the parameterization of the line of response (LoR) are introduced. This finally leads to a nonuniform and non-isotropic spatial resolution.

Different solutions have been proposed to reduce the parallax error: the use of scintillators with different decay times and pulse shape discriminators (PSD) [8] [7] [9] [10]; the stack of two or more crystal layers, each one set with an offset with respect to the one below [11]; the placement of photosensors at both ends of a crystal and use the ratio of the light from the two sensors to determine the DoI [12] and sharing the light between paired crystals to reflects the DoI [13].

An Analog to Digital Converter (ADC) is typically implemented in an ASIC design to process the signal obtained from the crystals. If the system has a moderated resolution requirement (as in PET systems), the ADC circuit can be replaced by a Time over Threshold (ToT) and a Time to Digital Converter (TDC). This implementation gains in simplicity, robustness and power consumption. ToT implementations have been widely used in particle physics and lately they are also gathering interest in medical imaging field.

In this work, we describe the FlexToT design for PET and the results obtained for the DOI problem with a phoswich detector. Section 2 details the FlexToT design for PET applications. Section 3 explains the setups used for the DOI measurements. Section 4 describes the algorithm to analyze the measurements and section 5 shows the experimental results obtained. Finally, section 6 closes with a summary and conclusions.

\section{FlexToT}

The FlexToT (Flexible Time Over Threshold) ASIC has been designed using AMS SiGe BiCMOS $0.35 \mu \mathrm{m}$ technology. It has 16 channels and each one is composed by an input current stage where the input current signal coming from an MPPC or SiPM is copied in three stages. For energy and pile-up stages the current is also scaled. The copied signals are transformed for time, energy and pile-up measurements into binary signals compatible with digital electronics such as FPGA.

The time stage is composed by a fast comparator which outputs the width of the input signal for a selected threshold. The energy stage contains the Time over Threshold (ToT) circuit composed by an integrator and a Schmitt trigger. The typical ToT design, composed by a charge amplifier, a shaper and a discriminator, has been replaced to obtain a linear Pulse Width Modulated (PWM) energy signal. An external Time to Digital Converter (TDC) implemented on an FPGA digitizes the energy output signals from the ASICs. The pile-up stage is composed by a differentiator and a fast comparator to discriminate events with more than one pulse. Figure 1 shows the block diagram 
of the FlexToT ASIC.

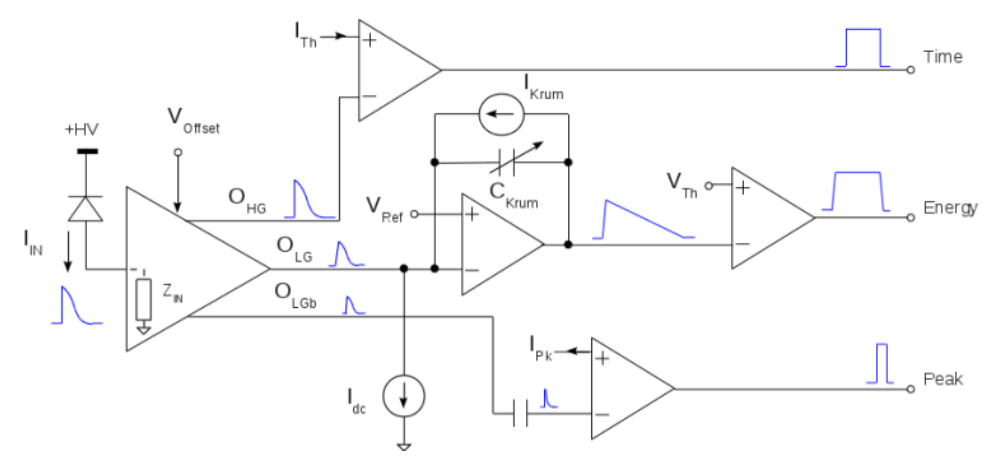

Figure 1: Block diagram of the FlexToT electronics.

Flexibility is one of the main characteristics of the design. The ToT circuit implementation presents the possibility of modifying a set of parameters to adapt to different scintillator crystals with different time constants or different sensors with a wide range of overvoltages. Figure 2 illustrates analog signal processing with the FlexToT ASIC, for two different input current signals with different time constant.
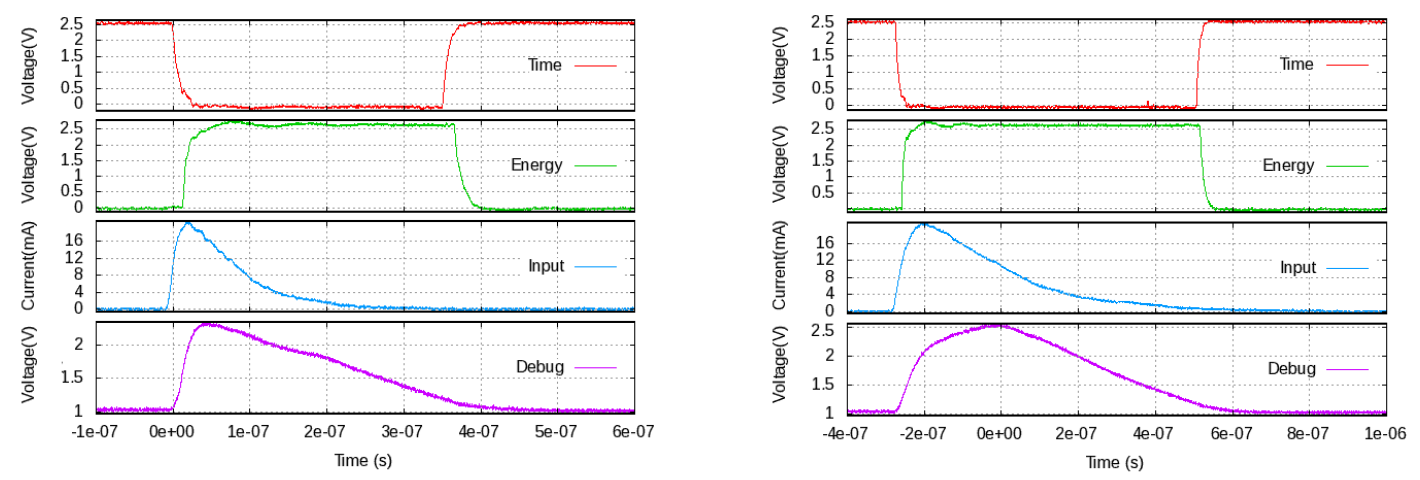

Figure 2: Typical analog signal processing with the FlexToT ASIC for different input signals with different time constants. The left plot shows the performance for a input signal with a $\tau=36 \mathrm{~ns}$, and the time constant of the right plot is $\tau=110 \mathrm{~ns}$. The figures in the first and the second row show the output signals in the time and energy stage. The 3rd row shows the input current signal and the 4th the signal obtained with an integration in the energy stage.

The transfer function of the energy path can be seen in Fig.3. The calibration measurements show the linearity performance for different peak currents and a linearity error below $\pm 5 \%$ almost for the whole range of input signals. The measurements were obtained using an electrical signal injected from a circuit developed to characterize the ASIC with an arbitrary waveform generator. 


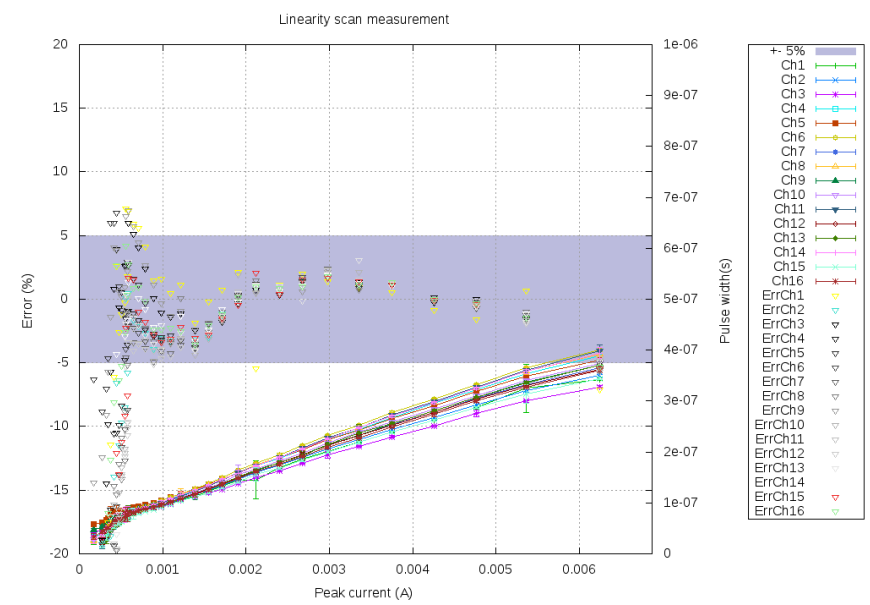

Figure 3: Transfer function of the energy path. The pulse width of the output signals (Y-right-axis) obtained for the corresponding current peak values in the $\mathrm{X}$-axis show the linear response obtained with the ToT stage. The linearity error (Y-left-axis) is less than the $\pm 5 \%$ in almost the whole response range.

\section{Setup}

Measurements using the phoswich detectors and the FlexToT read-out were performed in two different setups. The first experimental setup was composed by a small black box with one FlexToT board inside coupled to a 9x9 MPPC array and two scintillators crystals: one LYSO crystal of $1.35 \times 1.35 \mathrm{~mm}^{2}$ of section and $8 \mathrm{~mm}$ long and one GSO crystal with the same section and $7 \mathrm{~mm}$ long. A $100 \mathrm{kBq}^{22} \mathrm{Na}$ radioactive source was used to irradiate the detectors from their lateral side. This experiment was done in the Experimental HEP group facilities, in Barcelona.

Preliminary measurements made in this setup were focus on obtaining the response of the electronics to the input signal from each one of the crystals, separately. Figure 4 shows a schematics and a picture of this setup. As can be seen in the figure, the setup was composed by one FlexToT board biased with two power supplies. The first one, an Agilent E3631A, was used to bias the electronics board and the high voltage power supply. The second one, a Keithley SourceMeter 2612 was used to bias the MPPC. Measurements were performed using an oscilloscope Agilent Infiniium MSO9404A. Two probes were plug to the FlexToT board to obtain the energy and time responses. The whole system was controlled by a PC that set the bias voltages needed to polarize the system and provided the instructions to acquire the data from the oscilloscope.

After the preliminary measurements with separated crystals, a setup with stacked crystals was needed. This setup was build up in CIEMAT facilities and it is depicted in Fig.5. The goal of this setup was to measure the response of the system when a photon is absorbed by a known crystal. A lead collimator of $5 \mathrm{~cm}$ long and $7 \mathrm{~cm}$ of diameter was placed between the source and the crystals with the beam focused to the selected crystal. The collimator has a centered hole of $3 \mathrm{~mm}$ of diameter designed to collimate and focus the beam. A coincidence trigger using another FlexToT was set at a distance of $20 \mathrm{~cm}$ in the direction of the collimated beam to assure that the detected photon was detected by the aimed crystal. 

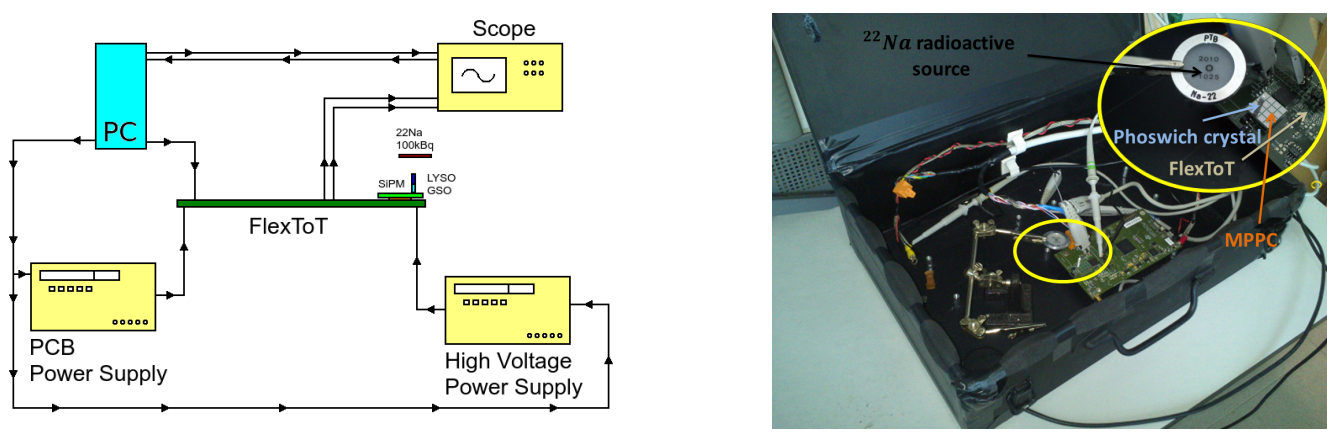

Figure 4: The left-most picture shows the setup schematic used in the University of Barcelona to perform the first measurements. The right-most picture shows a photograph of the small black box with the FlexToT board inside. The MPPC, the $22 \mathrm{Na}$ radioactive source and the phoswich crystals can be seen in the detailed enlarged region.

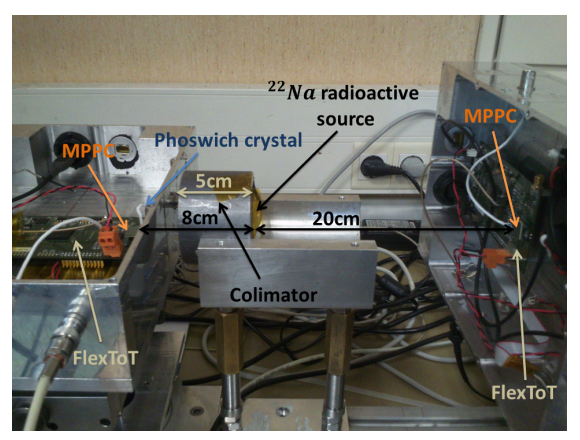

Figure 5: Setup used in CIEMAT facilities to measure the response of the FlexToT to a stacked phoswich crystal block. The basic idea was to collimate the beam from the radioactive source towards the selected crystal and assure a proper impinging direction using an electronic trigger.

\section{Algorithm for data analysis}

The analysis of the data acquired in the measurements with a phoswich detector requires data machine learning techniques [2] [5]. The rate of identification needs a supervised learning algorithm, that after a training using part of the measured data, it can classify input data in two sets, corresponding to the two stacked crystals. The classifier algorithm used for this experiment is the Support Vector Machine (SVM) [3]. A linear classifier based on the search of the dividing optimum hyperplane between samples was written. If the data sample, as in our case, is not linearly classifiable, a transform function called kernel can be used to transform the input data to a space in which the data is linearly separable. In this case, it was used the Radial Basis Function [4]. A gaussian transformation was used to obtain the optimum hyperplane. SVM algorithms not only can be used with non linearly separable data using kernels. A variation of the algorithm can be employed to process data non completely separable using the so-called soft margins.

Data measurements from the oscilloscope were used to feed directly the SVM algorithm in the first part of the analysis. An event selection algorithm was applied previously to the SVM to choose those below the $511 \mathrm{keV}$ peak of the energy distribution plot. In the next section we will present the results obtained for both analysis systems. 


\section{Measurements}

The working point for optimum signal separation is defined with the MPPC bias voltage and the time threshold. The energy resolution depends on the quantum efficiency of the MPPC; it degrades due to optical crosstalk. The time threshold defines the best separation of the time distributions. An example of the measurements obtained for time and energy distributions for different values of these parameters can be seen in Fig.6. As it is shown, the overlapping between distributions increases with the time threshold ${ }^{1}$ and the FWHM of the $511 \mathrm{keV}$ energy peak increases with the bias voltage. A summary of the results obtained can be seen in Fig.7.
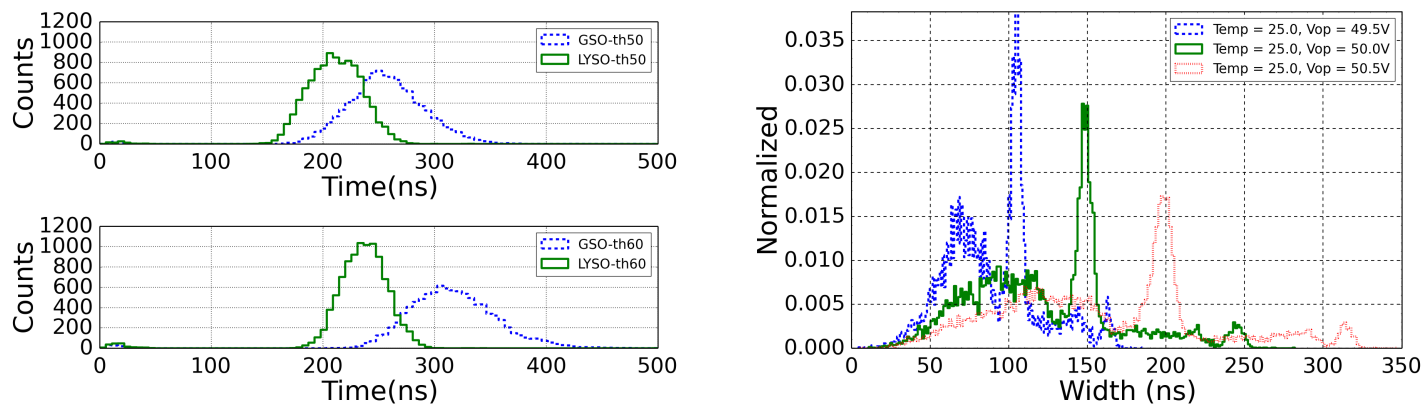

Figure 6: The left-most plots show the time distributions for two different thresholds. The right-most plot illustrates the energy distributions for different bias voltages.
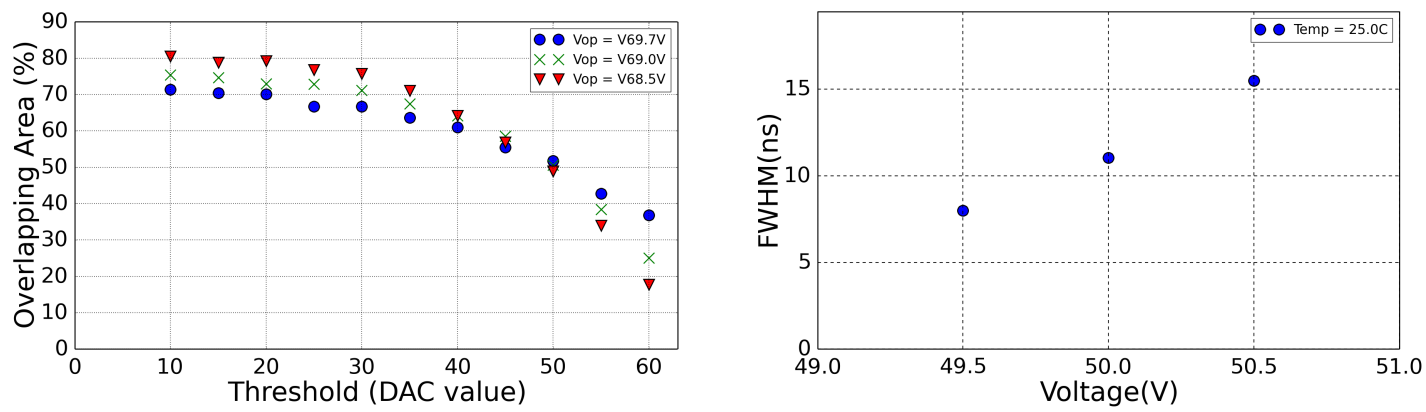

Figure 7: Summary of the key parameters to obtain the optimum working point for phoswich measurements.

First measurements were done without using stacked crystals. Figure 8 (left side) shows the 2D map of the energy versus time. It can be observed how the FlexToT ASIC distinguishes both crystals easily. The rate of identification obtained using the SVM algorithm without event selection was $98 \%$. Using the previous event selection algorithm the correct identification rate raised to $99 \%$. The rate of identification for crystals placed between two MPPC channels achieved a $98 \%$ without previous event selection.

\footnotetext{
${ }^{1}$ Threshold in FlexToT system is counted from the highest value (63) for the lowest threshold to the bottom value (0) for the highest threshold.
} 

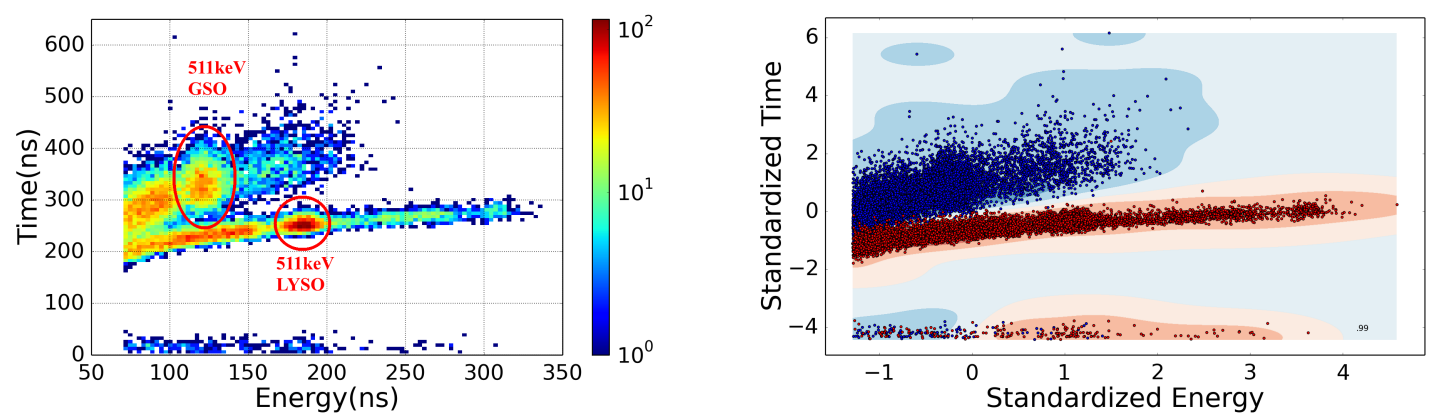

Figure 8: The left-most plot shows the time-energy distribution for separated crystals. The right-most figure depicts the analysis obtained with the SVM algorithm.

For an efficient evaluation of a stacked configuration of crystals, the setup illustrated in Fig.5 was required. In the used scintillator crystals, when the photon is absorbed, the generated electron range is around $200 \mu \mathrm{m}$. This short range causes that the pattern obtained from stacked crystals is the same than the one obtained from separated crystals. The main difference between separated and stacked crystals measurements is the stretching of both signals due to a reabsorption effect because of the double volume. Figure 9 shows precisely this effect of contraction compared to what is shown in Fig.8.

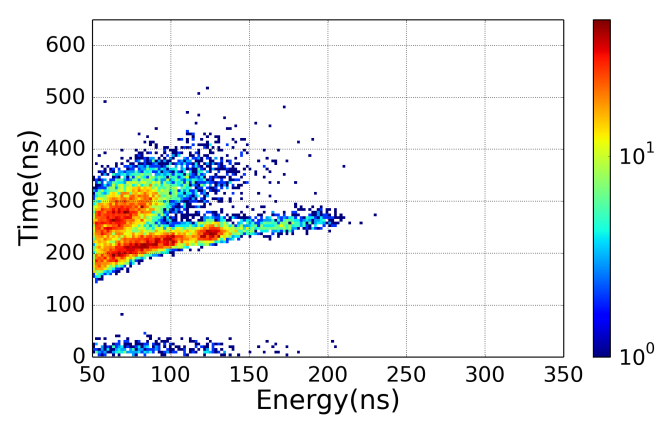

Figure 9: 2D energy-time map for stacked crystals.

\section{Conclusions}

FlexToT ASIC is a design for PET applications that employs electronics circuitry widely used in particle physics. The flexible configuration of the parameters of the energy ToT stage permits to employ the FlexToT for different scintillators with different decay constants and different SiPM with a wide range of bias voltages. This flexibility increases with the implementation of the TDCs in an FPGA.

A method to estimate the DoI in the FlexToT configuration has been tested, obtaining an excellent correct identification rate of $99 \%$ for separated LYSO and GSO crystals. Currently we are working to improve the experimental setups to obtain the identification rate for stacked crystals. The preliminary results of these tests are encouraging. 


\section{Acknowledgments}

We thank the company SEDECAL for supplying the scintillator crystals used in this research.

\section{References}

[1] D.Baley, D.W.Townsend, P.E.Valk, M.N.Maisey, Positron Emission Tomography, Springer, London, 2005

[2] T. M. Mitchell, Machine Learning, McGraw-Hill, New York, NY, 1997.

[3] N. Cristianini and J.Shawe-Taylor An Introduction to Support Vector Machines and Other Kernel-based Learning Methods, Cambridge University Press, 2000

[4] B. Scholkopf,K. K. Sung, C. J. C. Burges, F. Girosi, P. Niyogi, T. Poggio, and V. Vapnik, Comparing support vector machines with Gaussian kernels to radial basis function classifiers, IEEE Transactions on Signal Processing, 45(11):2758-2765 (1997)

[5] P. Domingos, A few useful things to know about machine learning, Commun. ACM 55, 10, 78-87 (2012)

[6] T. K. Lewellen, Recent developments in PET detector technology, Phys. Med. Biol. 53 R287 (2008)

[7] M. Streun, G. Brandenburg, G,H. Larue, H. Saleh, E. Zimmermann, K. Ziemons, H. Halling, Pulse shape discrimination of LSO and LUYAP scintillators for depth of interaction detection in PET, Nuclear Science Symposium Conference Record, 2002 IEEE, 1636 - 1639 vol.3 (2002)

[8] M. Dahlbom, L. R. MacDonald, L. Eriksson, M. Paulus, M. Andreaco, M. E. Casey, C. Moyers, Pulse shape discrimination of LSO and LuYAP scintillators for depth of interaction detection in PET, Nuclear Science, IEEE Transactions on 44, 3, 1114-1119 (1997)

[9] J. H. Jung, Y. Choi, Y. H. Chung, O. Devroede, M. Krieguer, P. Bruyndonckx, and S. Tavernier, Optimization of LSO LuYAP phoswich detector for small animal PET, Nucl. Instrum. Methods Phys. Res. A 571, 669-675 (2007)

[10] J. Seidel , J. J. Vaquero, S. Siegel, W. R. Gandler and M. V. Green, Depth identification accuracy of a three layer phoswich PET detector module, IEEE Trans. Nucl. Sci., 46, 485490 (1999)

[11] Nishikido F, Tsuda T, Inadama N, Yoshida E, Takahashi K, Shibuya K, Yamaya T, Kitamura K and H. Murayama, Spatial resolution measured by a prototype system of two four-layer DOI detectors for jPET-RD IEEE Nucl. Sci. Symp. Conf. Record (IEEE Cat. No. 06CH37832) pp 3041-4 (2006) 
[12] W. W. Moses,S. E. Derenzo,R. Nutt,W. M. Digby,C. W. Williams and M. Andreaco Performance of a PET detector module utilizing an array of silicon photodiodes to identify the crystal of interaction, IEEE Trans. Nucl. Sci. 40, 1036-40 (1993)

[13] T. K. Lewellen, M. Janes and R. S. Miyaoka DMice-a depth-of-interaction detector design for PET scanners IEEE Nucl. Sci. Symp. Conf. Record (IEEE Cat. No.03CH37515) pp 2388-92, (2004) 